\title{
The simple regularities in the dynamics of online news impact
}

\author{
Matúš Medo ${ }^{1,2,3}$ - Manuel S. Mariani ${ }^{1,4} \cdot \operatorname{Linyuan} \mathrm{Lü}^{1,5,6}$
}

Received: 14 March 2021 / Accepted: 19 August 2021 / Published online: 12 September 2021

(c) The Author(s) 2021

\begin{abstract}
Online news can quickly reach and affect millions of people, yet we do not know yet whether there exist potential dynamical regularities that govern their impact on the public. We use data from two major news outlets, BBC and New York Times, where the number of user comments can be used as a proxy of news impact. We find that the impact dynamics of online news articles does not exhibit popularity patterns found in many other social and information systems. In particular, we find that a simple exponential distribution yields a better fit to the empirical news impact distributions than a power-law distribution. This observation is explained by the lack or limited influence of the otherwise omnipresent rich-get-richer mechanism in the analyzed data. The temporal dynamics of the news impact exhibits a universal exponential decay which allows us to collapse individual news trajectories into an elementary single curve. We also show how daily variations of user activity directly influence the dynamics of the article impact. Our findings challenge the universal applicability of popularity dynamics patterns found in other social contexts.
\end{abstract}

Keywords Online information · Dynamics of impact · Collective attention · Evolving networks

Matúš Medo

matus.medo@unifr.ch

1 Institute of Fundamental and Frontier Sciences, University of Electronic Science and Technology of China, Chengdu 610054, People's Republic of China

2 Department of Radiation Oncology, Inselspital, University Hospital of Bern, University of Bern, 3010 Bern, Switzerland

3 Department of Physics, University of Fribourg, 1700 Fribourg, Switzerland

4 URPP Social Networks, Universität Zürich, 8050 Zurich, Switzerland

5 Complex Systems Lab, Beijing Computational Science Research Center, Beijing 100193, People's Republic of China

6 Yangtze Delta Region Institute (Huzhou), University of Electronic Science and Technology of China, Huzhou 313001, People's Republic of China 


\section{Introduction}

Consider a major news, like the results of the presidential elections or the onset of a global epidemic outbreak. In the 80 s, we would have discovered it through traditional print and broadcast media. Today, new media and online platforms have disrupted not only the way we discover and consume information, but also the way we form our opinions and attitudes about critical topics for our society like politics [1, $15]$, science [7, 24], and public health [25, 26]. Online newspapers and social media platforms are now the majour sources of information about events in the world [44] and provide us with rich data for the study of human attention [32, 52]. Despite the rise of social media, traditional newspapers and mainstream media are still important information sources with large audiences. The importance of mainstrean news sources can be illustrated by, for example, Facebook temporarily increasing their weight in the internal news ranking system in an attempt to respond to misinformation spreading following the U. S. presidential election. ${ }^{1}$

Most online newspapers allow users to directly comment on news articles [29], creating a "digital public sphere" where participation is free, recent events are publicly discussed, and comments are visible to everyone [42]. In such a complex information ecosystem, some news articles impact thousands of users who actively discuss and share them in online platforms, ${ }^{2}$ whereas many others remain little noticed. Therefore, understanding the dynamics of the impact of online news articles is vital not only because it deepens our understanding of how information spreads throughout modern societies, but also because it can potentially help to counteract negative side effects of new media like the spreading of misinformation [13, 49] and the amplification of ideological segregation [16].

The unprecedented availability of big data on human online activity has allowed us to uncover and model patterns of human behavior and cultural products' popularity in diverse contexts $[22,33]$, revealing universal regularities in the dynamics of cultural products as diverse as scientific papers [50], books [53], and songs [8], among others. As for online news articles, previous research has unveiled factors that make an online news article more likely to become popular, including story topic [9], content emotion [5], perceived objectivity [30], and format [34]. Yet, we do not know yet whether there exist universal regularities that govern the dynamics of online news articles' impact. Does the impact of online news articles follow similar patterns as the impact of other types of information items? Is article impact broadly distributed? Are there universal impact patterns for online news articles? How predictable is the dynamics of attention decay for online news articles? To address these questions, we analyze a novel dataset that contains commenting sections of 3087 articles from the British Broadcasting Corporation (BBC) and a dataset that contains commenting sections of 2801 articles from the New York Times (NYT).

\footnotetext{
${ }^{1}$ https://www.theverge.com/2020/11/24/21612728/facebook-news-feed-us-election-change-mainstreamnews-misinformation.

2 https://www.nytimes.com/2017/12/20/insider/our-most-commented-on-articles-of-2017.html.
} 
Previous works have generated insights that generalize well across domains: popularity and impact typically follow heavy-tailed distributions, leading to the emergence of a small number of "hits" [3,46] with disproportionate popularity. These successful outliers emerge from a combination of quality (often referred to as fitness) and social amplification mechanisms such as the rich-get-richer phenomenon [36]. These regularities in popularity dynamics have been found to govern the popularity and impact dynamics of cultural items as diverse as scientific papers $[36,50]$, websites [27], books [53], and patents [20, 21], among others.

Surprisingly, we find none of these regularities in the impact of online news. Differently from the widespread heavy-tailed distributions of popularity and impact in social systems, news impact in terms of the number of received comments) is exponentially distributed. Different categories of news have widely different average comment counts, yet their distributions can be collapsed onto a universal exponential distribution. The exponential impact distribution results from the absence or saturation of the widely-studied preferential attachment mechanism. In line with recent findings on the attention decay in science and technology [8, 20], the decay of individual news articles follows a universal exponential form. The impact dynamics of online news articles can be reproduced by a parsimonious model with articlelevel fitness and exponential aging [18]. Building on this model, we can predict the articles' long-term impact based on early activity. We study the impact of natural daily variations of user activity on the dynamics of the article impact and formulate a generalized dynamical model which includes the overall level of user activity as an additional factor along with article fitness and an aging term.

Our findings contribute to the literature on popularity dynamics $[17,22,27,36$, 50,53 ] by demonstrating that there is a limit to the generality of widely-observed patterns and mechanisms (such as preferential attachment). While previous studies have emphasized the generality of observed patterns of popularity and impact [8], future research might put more emphasis on identifying violations of pervasive patterns and the causes behind the observed violations. Besides, as managing and influencing the spreading of online information is vital for online newspapers and social platforms, our models and methods can be used to inform decisions by newspaper editors and content creators.

\section{Results}

\section{News impact is exponentially distributed}

By writing comments, the users demonstrate a higher level of engagement compared to only reading the article $[29,30]$. Importantly, comments are read also by users who do not actively comment, indicating that they play an important role in how a news article is perceived by the public [4]. The number of comments can be thus considered as a useful proxy for the article impact [47]. To study the distribution of article impact, we discard potential multiple comments from a single user on a given news, thus counting the number of unique users commenting on each article. When all comments are used instead, the results do not change qualitatively (see 
Supplementary Information, SI, for the results). We further benefit from the additional category information provided directly by the news outlets for all news; the most populated categories are football (BBC) and national (NYT); see Tab. S1 in the SI for details. Since comment counts strongly depend on the category of the news, we analyze news impact individually for each category.

How is the article impact distributed? Impact distributions for creative works are typically found to be heavy-tailed: this is the case for scientific papers [36], patents [48], and books [53], among others. Broad popularity distributions are also typically found for user-generated content in online systems [10]. Based on these findings, one might expect that the article impact too follows a heavy-tailed distribution. Surprisingly, we find instead that the distributions exhibit exponential tails for both BBC and NYT data. Using the exponential distribution ${ }^{3} P(c) \sim \exp (-\lambda c)$ for $c \geq c_{\min }$ and following the methodology introduced in Ref. [11], we obtain estimates for the lower bound $\hat{c}_{\min }$ and the scaling parameter $\hat{\lambda}$, together with the $p$ value obtained through the Kolmogorov-Smirnov test (see "Methods" section for details).

For football BBC news, we find that the exponential tail of the distribution begins at $\hat{c}_{\text {min }}=381$ and comprises 284 articles (18\% of all football articles). Ignoring a single outler with 3538 comments (see Sec. S2 in the SI for information on outlier detection), the estimated scaling parameter is $\hat{\lambda}=270 \pm 17$ and the high $p$ value of 0.96 indicates that the exponential distribution cannot be ruled out. The good fit can be visually appreciated by observing that the empirical distribution lies within the 5th-95th percentile range of synthetic exponentially distributed data generated with the estimated parameters (Fig. 1A). For national NYT news, the estimated lower bound is even lower, $\hat{c}_{\min }=28$, and the $p$ value is 0.53 (Fig. 1C) which again means that an exponential distribution is plausible. Detailed fitting results for all 20 news categories with at least 100 news are shown in the SI, Sec. S2. Importantly, the identified exponential tails are substantial, comprising more than $90 \%$ of news for 10 out of 20 analyzed news categories. The log-likelihood test [11] shows that an exponential distribution fits the data better than a power-law distribution for all categories but one (Learning in the NYT data, see Sec. S2.1 in the SI for additional information).

Inspired by the universality of scientific impact distributions [17, 39], we explore an intriguing possibility: By leveraging the estimated parameters, can we collapse the article impact distributions for different categories on top of each other? We find that this is the case: impact distributions in different categories collapse on top of each other after the comment counts are transformed as $\left(c-\hat{c}_{\min }^{X}\right) / \hat{\lambda}^{X}$, where $\hat{c}_{\min }^{X}$ and $\hat{\lambda}^{X}$ are the estimated lower bound and the scaling parameter for category $X$.

In summary, we find the impact distributions in individual news categories to be far from being power laws. Simple exponential fits work well in several categories where they describe the impact of a majority of news with remarkable veracity.

\footnotetext{
${ }^{3}$ Strictly speaking, $P(c)$ should be referred to as a geometric distribution as $c$ is a discrete variable.
} 

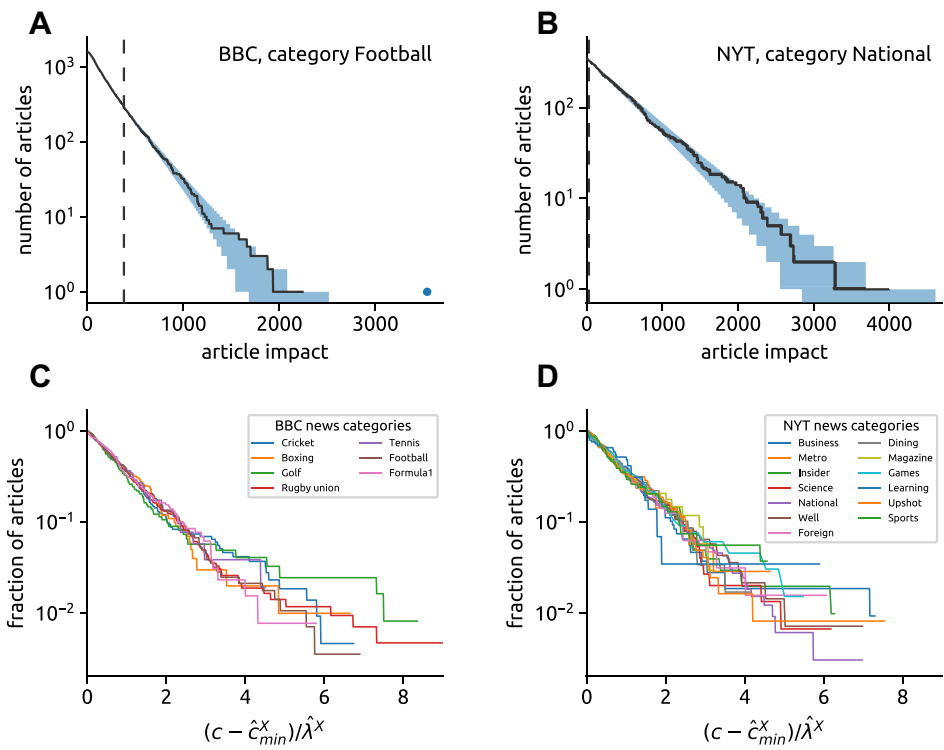

Fig. 1 Article impact is exponentially distributed. A, B Comment count distributions for football news in the BBC data and national news in the NYT data. For the football category, the dot shows a single outlier that was identified in the exponential fitting. C, D The distribution of the transformed comment counts, $\left(c-\hat{c}_{\min }^{X}\right) / \hat{\lambda}^{X}$, in individual news categories; here $\hat{c}_{\min }^{X}$ and $\hat{\lambda}^{X}$ are the exponential distribution parameters for category $X$. Upon rescaling, a universal distribution of article impact emerges

\section{The relation between article impact and user degree}

The observed article impact distributions, albeit narrower than in many other technosocial systems, still comprise articles that have much greater audience than most other articles. This gives us the possibility to study the relation between impact of an article and degree of the users who have commented on this article. Such a relation can exist if, for example, little active users are mostly idle and comment only on high-impact articles; such a connection would in turn contribute to the high impact of those articles. To assess the level of degree assortativity in the bipartite articleuser network, we divide both articles and users in five groups by their degree in such a way that the total degree in each group is approximately the same; groups 1 and 5 have the lowest and the highest degree users/articles, respectively. We then count the numbers of links between respective user and article groups and divide them with the average numbers of links observed in randomized networks. The resulting link propensity quantifies how much more likely (if propensity is above one) or less likely (if propensity is below one) are links between a given user and item group compared to a randomized network. For network randomization, we use the recently introduced Dynamic Configuration Model (DCM, [40]) which is a version of the classical configuration model for networks that grow in time. The DCM internally divides the network in $L$ layers and $L$ is a parameter of the model. Due to the quick aging that we observe in the analyzed datasets (see the following sections), we chose the number of layers to be the same as the number of days in each respective dataset 


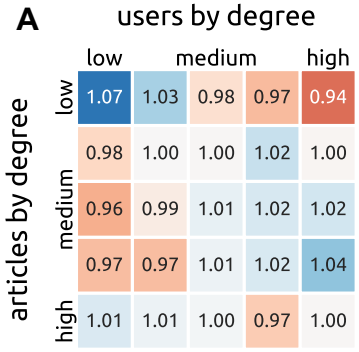

BBC data

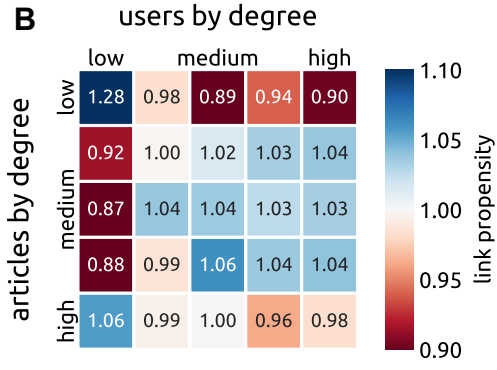

NYT data

Fig. 2 Relative link propensity between users and articles of various degree. Both users and articles are divided in five groups by their degree. The relative link propensity values quantify the excess (values greater than one) or lack (values smaller than one) of links between the respective pair of user and article groups as compared to randomized networks (we average over 1000 realizations of the DCM model [40]). The left and right panels show the results for the BBC data and the NYT data, respectively. While some statistically significant deviations from the null model can be observed (values that differ from one by more than, approximately, 0.02 have absolute $z$-scores above 3 ), only one of them (links between lowdegree users and low-degree articles in the NYT data) is larger than $20 \%$ either way

(the results are robust with respect to the choice of $L$ ). See Ref. [37] for a principled way to determine $L$ in a monopartite growing network.

Figure 2 shows that the relative link propensity is close to one for most pairs of user and article groups. One emerging pattern shared by the BBC and NYT data concerns the least popular articles which are commented by the least active users more than expected and by the most active users less than expected. By contrast, the original hypothesis of the most popular articles owing their popularity to little active users is ruled out by the results. For the BBC data, the relative link propensity between the most popular articles and the least active users does not differ significantly from one. For the NYT data, it is only 1.06 which means that the most popular articles do not receive $20 \%$ of their comments from the group of least active users who write $20 \%$ of all comments but $20 \% \times 1.06 \approx 21 \%$ which is a negligible increase. We can conclude that the most popular articles receive comments from users of all degree values approximately in line with expectations.

\section{Preferential attachment plays a minor role in the dynamics of impact}

The empirical exponential distributions of article impact inevitably lead us to investigate possible mechanisms behind their emergence. Motivated by existing results on the dynamics of impact for cultural products as diverse as scientific papers [36, 50], patents [20], and bestseller books [53], one expects two main forces shaping the dynamics of news impact [8]: preferential attachment and temporal decay. We start by addressing preferential attachment which implies that the rate at which article $i$ receives new comments, $\Delta c_{i}(t) / \Delta t$ where $\Delta c_{i}(t)=c_{i}(t+\Delta t)-c_{i}(t)$, is a power-law function (most commonly, a linear function) of the number of already-received comments, $c_{i}(t)$. 

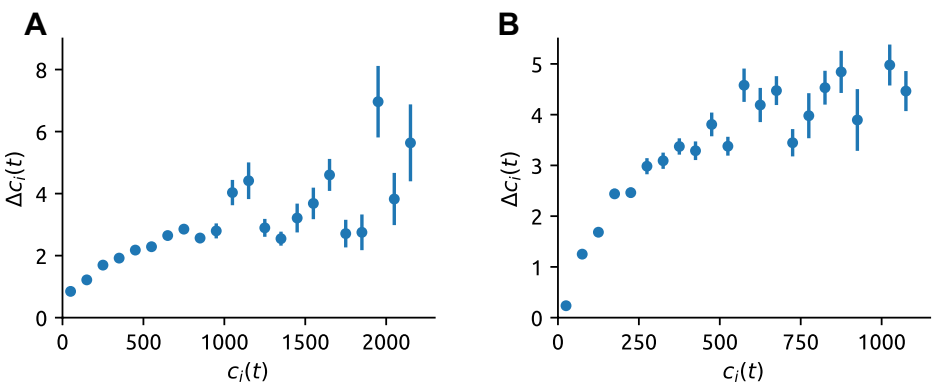

Fig. 3 Preferential attachment in the dynamics of article impact. The number of new comments in $\Delta t=10 \mathrm{~min}$ as a function of the current number of comments. For the BBC data (A), the fit up to the comment count 800 yields the slowly-growing dependence proportional to $1+c_{i}(t) / 220$. Above 800 comments, the dependence is even weaker (saturation). For the NYT data (B), sublinear preferential attachment with the exponent 0.79 is the best fit, followed by saturation for $c_{i}(t) \gtrsim 400$. Error bars indicate the standard error of the mean

In contrast with pervasive findings in the popularity dynamics literature, we find that preferential attachment is negligible in the BBC data (Fig. 3A) and exhibits clear sub-linearity and saturation for the NYT data (Fig. 3B). More specifically, in the BBC data, more than 200 comments are needed for an article to double its commenting rate with respect to a comment-free article. Furthermore, the observed weak growth of $\Delta c_{i}(t)$ with $c_{i}(t)$ can be explained in terms of a dynamic model where no preferential attachment is present (see Fig. S9 in the SI). In the NYT data, $\Delta c_{i}(t)$ first grows as a power of $c_{i}(t)$ with an exponent below one (sub-linear preferential attachment [28], see Fig. S10 in the SI) and becomes independent of $c_{i}(t)$ for $c_{i}(t) \gtrsim 400$. The lack of/saturation of preferential attachment has important consequences as it prevents a power-law degree distribution from emerging (see Section on modeling the article impact dynamics and Sec. S4 in the SI). In summary, we find that despite the articles' comment counts explicitly reported by both BBC and NYT (see Fig. S1 in the SI), the impact of preferential attachment on the dynamics of news article impact is limited.

\section{The dynamics of article impact follows an exponential decay}

Existing studies have found various functional forms for the decay of the impact of cultural items, including power-law [12], log-normal [50, 53], exponential [20, 38], stretched exponential [51], and biexponential [8]. To quantify the temporal decay of article impact, for each news $i$, we measure the news' number of new comments relative to the article's final comment count, $f_{i}(t):=\Delta c_{i}(t) / c_{i}$, as a function of the article age, $t$. The normalization by the article's final comment count makes the dynamics of articles of different ultimate impact directly comparable. ${ }^{4}$ For each

\footnotetext{
4 This normalization is also motivated by the observation that under negligible preferential attachment, $\Delta c_{i}(t) / c_{i}$ is expected to accurately capture the aging function of the articles, as shown in the next section.
} 

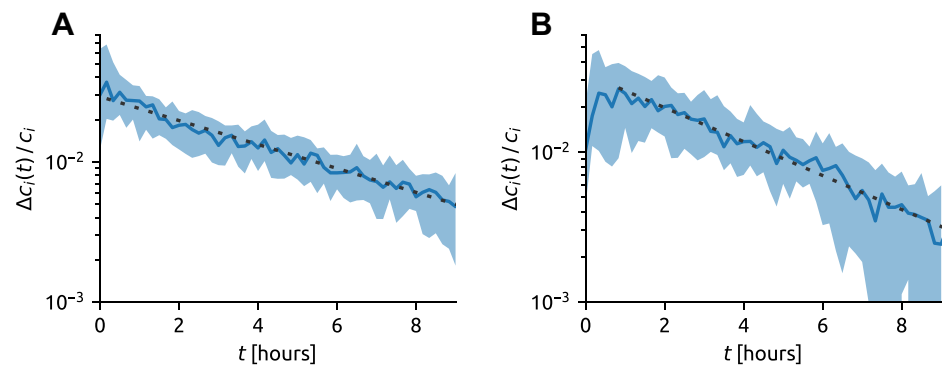

Fig. 4 Aging in the dynamics of article impact. The number of new comments of an article, $\Delta c_{i}(t)$, normalized by the final number of comments, $c_{i}$, as a function of its age, $t$, for the hit articles (90th percentile by the number of comments). The dotted lines indicate the linear fit for age $0-10 \mathrm{~h}$; their slopes correspond to representative aging timescales $\Theta=305 \mathrm{~min}(\mathrm{BBC}$, panel $\mathbf{A}$ ) and $\Theta=230 \mathrm{~min}(\mathrm{NYT}$, panel B), respectively. The time-of-day effects are suppressed here by including only the articles that appear in the morning between 9 am and noon (BBC) and between 2 pm and 5 pm GMT (NYT). The shaded areas there indicate the 20th-80th percentile range and the solid lines show the median values for the considered articles

age, $t$, we compute the median of $\Delta c_{i}(t) / c_{i}$ over all considered articles, obtaining the representative decay function, $f(t)$. We restrict the analysis to hit articles which, for the purpose of this work, are defined as the articles whose number of comments is above the 90th percentile (679 and 428 comments in BBC and NYT, respectively). To suppress the time-of-day effects, we include only the BBC articles that appeared in the morning between 9 am and noon-the $10 \mathrm{~h}$ range shown in Fig. 4A is thus a period when user activity is rather uniform at the BBC website. User activity is substantially lower in the night, which directly effects the evolution of $c_{i}(t)$ (see Sec. S3 in the SI for more details). For the same reasons, we focus on the NYT articles that appeared between $2 \mathrm{pm}$ and $5 \mathrm{pm}$ GMT.

We find that the articles' temporal decay follows a universal exponential form (Fig. 4). In particular, the average decay function $f(t)$ can be accurately fitted by an exponential function: $f(t)=\mathrm{e}^{-t / \Theta}$ where $\Theta=305 \mathrm{~min}$ for BBC and $\Theta=230 \mathrm{~min}$ for NYT. While $f(t)$ decreases exponentially in the BBC data during the whole observed range, it shows a short period (approximately $1 \mathrm{~h}$ ) of increase in the NYT data. This is a direct consequence of the preferential attachment that applies for low comment counts-as the number of comments grows, the rate of commenting initially accelerates before aging in combination with sublinear/saturated preferential attachment eventually cause the rate of commenting to decrease.

Our finding of a regular exponential decay of article impact agrees with the report of real news on Twitter differing from rumors by exhibiting a regular pattern of a monotonous decrease of attention [31]. The observed exponential aging can be explained by a simple model where each article is of interest to a fixed pool of readers (the pool's size is determined by the article's attractiveness to the readers) and every reader has a fixed probability to write their comment (most readers comment in a discussion only once) per time unit [23]. The observed exponential decay can be also interpreted as a limit scenario of the bi-exponential impact decay predicted by a recent work based on a model with communication memory and cultural memory 
[8]. The reason why such a limit scenario holds for online news needs to be clarified by future research. A plausible hypothesis is that as the comments to online news articles unfold over a narrow time period following a news, we cannot use them to observe the process whereby the communication memory associated with an article is converted into cultural memory. If this is the case, the model in [8] predicts an exponential decay of collective attention, in line with our observed decay functions.

\section{Exponentially distributed fitness and exponential aging shape the dynamics of article impact}

The impact dynamics for scientific papers $[36,50]$ and bestseller books [53] is typically modeled in terms of preferential attachment, fitness and aging. Building on these studies, a potential model for the commenting dynamics would assume that the expected rate at which article $i$ receives new comments at time $t$ is

$$
\Delta c_{i}(t) / \Delta t=\left[1+c_{i}(t)\right] \eta_{i} f_{i}\left(t-t_{i}\right)
$$

where $1+c_{i}(t)$ is the preferential attachment factor, $\eta_{i}$ is the fitness factor, $f_{i}\left(t-t_{i}\right)$ denotes an article-dependent aging function, and $t_{i}$ is the appearance time of article $i$. In line with previous studies [36, 50], article fitness $\eta$ is a hidden intrinsic parameter that quantifies, other factors being equal, how a given article is attractive to the website's audience. We refer to this model as the PFA model because it includes Preferential attachment, Fitness and Aging. In this model, a narrow exponential distribution of article fitness, $\rho(\eta)=\exp (-\eta)$, leads to the emergence of a power-law distribution of the comment count [36]. In other words, small differences in items' fitness are amplified by preferential attachment and produce wide impact inequalities.

The observed weak preferential attachment and exponential temporal decay suggest a simpler model of the dynamics of article impact where only article fitness and exponential aging play a role. We thus assume that the rate at which article $i$ receives new comments at time $t$ is

$$
\Delta c_{i}(t) / \Delta t=\eta_{i} f_{i}\left(t-t_{i}\right)
$$

which we refer as the FA (Fitness-Aging) model [18]. To accurately represent the commenting dynamics, we introduce individual aging timescales $\Theta_{i}$ and the aging factor in the form $f_{i}\left(t-t_{i}\right)=\exp \left[-\left(t-t_{i}\right) / \Theta_{i}\right]$. The aging timescales $\Theta_{i}$ are estimated from the empirical data by minimizing the Kolmogorov-Smirnov statistic between the comment count dynamics in the model and in the empirical data (see Sec. S6 in the SI). If $\Theta_{i} \gg 1$, the expected final comment count under the FA model is directly proportional to the product of the article fitness and the aging timescale, $\overline{c_{i}}=\eta_{i} \Theta_{i}$ (see Sec. S5 in the SI). The model further implies that

$$
\frac{\overline{c_{i}(t)}}{\overline{c_{i}}}=1-\exp \left(-\frac{t-t_{i}}{\Theta_{i}}\right) .
$$



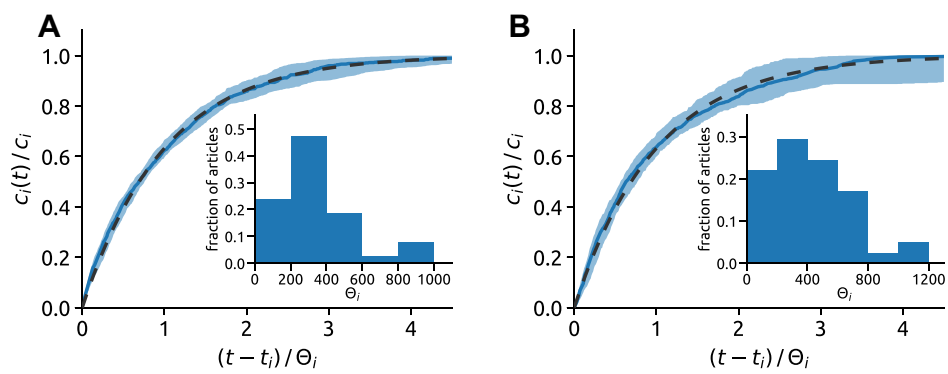

Fig. 5 The universal dynamics of article impact. The comment count evolution in terms of the normalized article age $\left(t-t_{i}\right) / \Theta_{i}$ for the BBC (A) and NYT (B) data. The shaded areas there indicate the 20th80 th percentile range and the solid lines show the median values for the considered articles. The dashed line represents the proposed model and its solution given by Eq. (3). The inset shows the distribution of the timescales $\Theta_{i}$ obtained by minimizing the Kolmogorov-Smirnov statistic. Only "morning" articles are included in the analysis, as in Fig. 4

Motivated by this result, we measure the dynamics of the comment count normalized by the final comment count. We find that Eq. (3) captures the empirical dynamics remarkably well (Fig. 5) and allows us to collapse all article trajectories onto a universal curve (Fig. 5). This result demonstrates that the fitness-aging model captures the two essential factors that govern the dynamics of news article impact, and it further confirms that preferential attachment plays a negligible role in the emergence of hit articles. Combined with insights on empirical user dynamics (see Fig. 2 and Sec. S3 in the SI), Eq. (2) can be also formulated in terms of a growing bipartite network where $\Delta c_{i}(t) / \Delta i$ is the rate at which article node $i$ attracts new links.

Since $\overline{c_{i}} \sim \eta_{i} \Theta_{i}$, exponentially distributed $\eta \Theta$ leads to the emergence of an exponential comment count distribution in line with the empirical data. When the aging timescales vary relatively little among the articles, as is the case here, the distribution of article fitness alone is approximately exponential. Interestingly, an exponential distribution of $\eta \Theta$ (referred to as total relevance therein) was reported in Ref. [36] for scientific papers and an exponential distribution of $\eta$ (in a model without aging) was reported in Ref. [27] for pages of the world wide Web. To identify theoretical mechanisms behind this widespread emergence of exponentially distributed fitness of items remains an important future challenge.

\section{Early activity can be used to predict article impact}

The regular dynamics demonstrated by Fig. 5 suggests that the early commenting activity and the final article impact are highly correlated. To verify this conjecture, we study a classification problem where we aim to predict whether an article will become a hit (i.e., if it will belong to the 90th percentile by the final impact). We classify an article as positive if it belongs to the 90th percentile by the number of comments that it has attracted over the first $\Delta t$ minutes, and negative otherwise. We evaluate the classifier using precision and AUC which are both classical information retrieval metrics [35] that range from zero (the worst result) to one (the best result). We find that the proposed simple classifier exhibits high values of precision 
Table 1 Classification precision and AUC for the hit articles

\begin{tabular}{llllll}
\hline & \multicolumn{2}{l}{ BBC data } & & & \multicolumn{2}{l}{ NYT data } \\
\cline { 2 - 3 } \cline { 6 - 6 }$\Delta t$ & $P$ & AUC & & $P$ & AUC \\
\hline 1 & 0.33 & 0.69 & & 0.31 & 0.62 \\
2 & 0.51 & 0.81 & & 0.51 & 0.75 \\
5 & 0.60 & 0.87 & & 0.64 & 0.89 \\
10 & 0.63 & 0.89 & & 0.73 & 0.92 \\
60 & 0.69 & 0.92 & & 0.81 & 0.96 \\
240 & 0.77 & 0.94 & & 0.83 & 0.98 \\
1200 & 0.93 & 0.99 & & 0.95 & 0.99 \\
\hline
\end{tabular}

and AUC even when $\Delta t$ is short: precision exceeds 0.6 after $5 \mathrm{~min}$, for example (see Table 1 for full results).

The observed predictability is unsurprising given previous results on the correlation between early and late popularity of online content [10, 43, 45]. However, previous studies interpreted the early-stage predictability of the virality of online cascades as a possible manifestation of cumulative advantage [43]. This cannot be the case here for online news where we demonstrated that preferential attachment has a negligible effect. Taken together, our findings suggest a somewhat simpler scenario: the news that are highly attractive for the public tend to receive more connections throughout their whole lifetime than less attractive news. In this sense, the impact of online news might be seen as more "meritocratic" than that of content in systems with preferential attachment: The news with truly high fitness are those that eventually succeed, regardless of cumulative advantage effects.

\section{Circadian patterns of user activity patterns shape the dynamics of news impact}

To study the impact dynamics, we have until now focused specifically on "morning" articles that benefit from high and approximately constant user activity for more than $10 \mathrm{~h}$ after their publication. We now turn our attention to the effect of overall user activity, which naturally decreases in the night (see Fig. S7 in the SI), on the dynamics of article impact. Figure 6A shows the evolution of the median number of new comments for the same set of morning articles over a longer period. This allows us to observe a decrease of commenting activity in the night (age 12-18 h) and renewed exponential decay on the second day (age 20-29 h) with the timescale of $312 \mathrm{~min}$. The two fitted exponential decay timescales, $302 \mathrm{~min}$ for article age 0-10 $\mathrm{h}^{5}$ and $312 \mathrm{~min}$ for article age 20-29 h are remarkably close to each other. We see that after user activity recommences after a night, article aging continues in the same speed than before the night.

\footnotetext{
5 The minor difference with respect to Fig. 4 where the fitted timescale is 305 min is due to the use of wider bins in Fig. 6A to improve the statistics for high article age.
} 

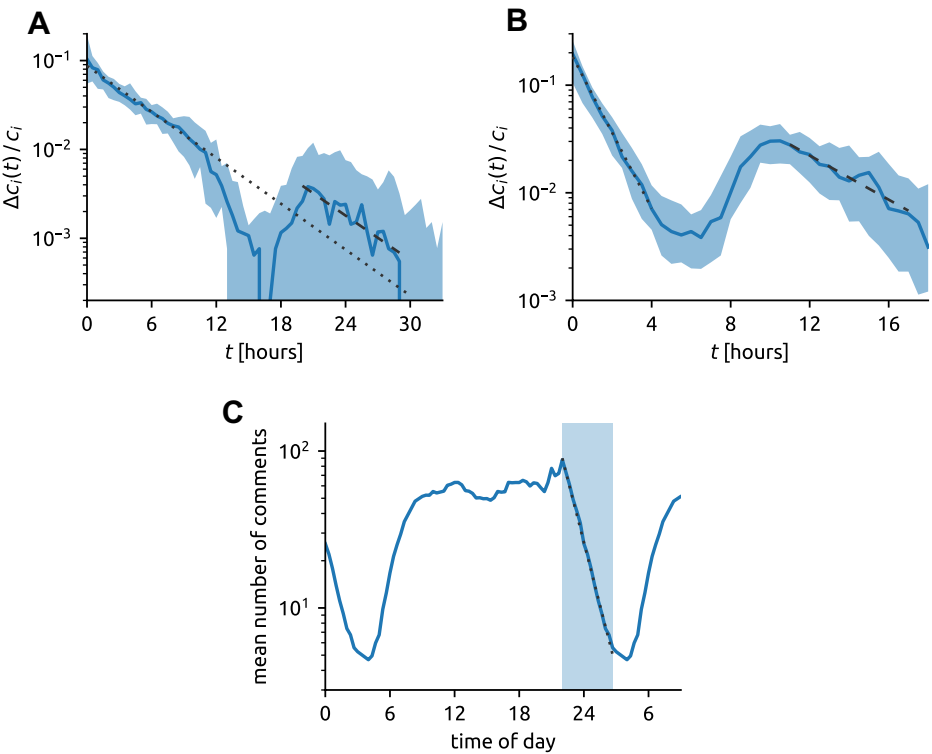

Fig. 6 Interplay between exponential aging and circadian user activity patterns in the BBC data. A The number of new comments of an article, $\Delta c_{i}(t, \Delta t)$, normalized by the final number of comments, $c_{i}$, as a function of its age, $t$, for morning hit articles (published between 9 am and noon). The dotted line indicates the linear fit for age $0-10 \mathrm{~h}$ (fitted timescale $302 \mathrm{~min}$ ). The dashed line indicates the linear fit for age 20-29 h (fitted timescale $312 \mathrm{~min}$ ). B As (A) for evening articles (published between $9 \mathrm{pm}$ and midnight). Timescales of the indicated fits are $74 \mathrm{~min}$ (first $4 \mathrm{~h}$, dotted line) and $254 \mathrm{~min}$ (age 11-17 h, dashed line). In panels (A) and (B), the age bin size is $30 \mathrm{~min}$ to achieve better statistics for high article age. $\mathbf{C}$ The course of the mean number of comments in 20 min intervals during the day at the BBC Sport website. The fitted timescale of the exponential decrease between $10 \mathrm{pm}$ and $3 \mathrm{am}$ is $97 \mathrm{~min}$

Figure $6 \mathrm{~B}$ shows the evolution of the median number of new comments for evening hit articles (articles published between $9 \mathrm{pm}$ and midnight). We see again two phases of exponential decay: an early phase during the night (age up to $4 \mathrm{~h}$ ) with the timescale of $74 \mathrm{~min}$ and a late phase during the day (age 11-17 h) with the timescale $254 \mathrm{~min}$. Albeit having a somewhat shorter timescale, the late phase is a direct equivalent of the previously observed aging of morning articles. We thus see that morning and evening articles exhibit similar aging during the day.

On the other hand, the early night aging of evening articles has a timescale which is significantly shorter than the aging timescle observed during the day. To better understand this fast aging, Fig. 6C shows the average overall activity on the BBC Sport website. We see that after an approximately constant activity during the day (from 8 am until $8 \mathrm{pm}$ ) and a small peak in the evening (from 8 pm until $10 \mathrm{pm}$ ) when many sport events take place, user activity dramatically decreases after $10 \mathrm{pm}$. Moreover, the user activity decay during the first $5 \mathrm{~h}$ of the decrease (from $10 \mathrm{pm}$ until $3 \mathrm{am}$ ) is a nearly perfect exponential with the fitted timescale of $97 \mathrm{~min}$. Varying user activity can be included in the previously introduced FA model described by Eq. (2) by introducing it as an additional multiplicative 
factor, thus obtaining an FAA (Fitness-Aging-Activity) model. The rate at which article $i$ receives new comments at time $t$ then reads

$$
\Delta c_{i}(t) / \Delta t=\eta_{i} f_{i}\left(t-t_{i}\right) A(t)
$$

where $t_{i}$ and $\eta_{i}$ are the appearance time and fitness of article $i$, respectively, and $A(t)$ is the overall activity factor which is common to all articles at the platform. If we now assume that article $i$ appears when user activity, $A(t)$, decreases exponentially, we obtain

$$
\Delta c_{i}(t) / \Delta t=\eta_{i} \exp \left[-\left(t-t_{i}\right) / \Theta_{A}\right] A\left(t_{i}\right) \exp \left[-\left(t-t_{i}\right) / \Theta_{U}\right]
$$

where $\Theta_{A}$ and $\Theta_{U}$ are the article and user exponential decay timescales, respectively. The two exponential terms can be combined in one as

$$
\exp \left[-\left(t-t_{i}\right) / \Theta_{A}\right] \times \exp \left[-\left(t-t_{i}\right) / \Theta_{U}\right]=\exp \left[-\left(t-t_{i}\right) / \Theta_{J}\right]
$$

where the joint exponential timescale, $\Theta_{J}$, has the form

$$
1 / \Theta_{J}=1 / \Theta_{A}+1 / \Theta_{U}
$$

Using the fitted values $\Theta_{A}=302 \mathrm{~min}$ and $\Theta_{U}=97 \mathrm{~min}$, we obtain $\Theta_{J}=73 \mathrm{~min}$ which is in an excellent agreement with the fitted aging timescale 74 min of evening articles during the night. Results are qualitatively similar for the NYT data (see Sec. S7 in the SI). We can thus conclude that the FAA model given by Eq. (4) presents an effective way of combining article dynamics with circadian and other patterns of varying user activity.

\section{Discussion}

By analyzing data on the comments to online news articles in two major nationwide newspapers, we were able to uncover surprising empirical regularities that characterize the distribution of the impact of online news articles and the impact dynamics. In particular, we revealed two universal patterns: (1) For both newspapers, the distribution of the number of comments received by articles from various categories collapse onto a universal exponential curve and (2) the dynamics of the comment count of different news articles collapse onto a universal curve once appropriate rescaling is applied. The exponential impact distribution emerges from impact dynamics where preferential attachment plays a negligible role. This indicates that differently from other social systems $[41,51]$, popularity signals in online newspapers are not prominent enough to play a significant role. Main empirical dynamical patterns of article impact can be reproduced with a minimal model which combines article fitness, an aging term (which in our case has an exponential form), and overall user activity. When user activity is approximately constant, only article impact and aging remain and the resulting dynamics is particularly simple.

Our findings contrast with the previous literature on success and popularity that has emphasized that success and popularity are usually characterized by heavy-tailed 
distributions [6, 10, 27, 39], and that preferential attachment plays a key role in shaping the emergence of hits $[27,36,50,53]$. Additional research is needed to quantify the relative importance of different factors that trigger user engagement in a news article (i.e., which article attributes contribute to its fitness $[2,5]$ ), and how our findings generalize to different cultures and platforms in languages other than English.

We quantified the impact of a news article through the number of comments it received from the online newspaper's readers. Other metrics of impact might be also relevant to news outlets. For example, the overall impact of a news can be quantified as a combination of the impact on the readers of the newspaper and the impact on users who shared or commented the news in different social media and news aggregation platforms. Uncovering the regularities of the news articles' dynamics by incorporating data from social media and news aggregators is an important direction for future research, given the critical role of these platforms for news dissemination $[14,19]$.

Although our study focused on news outlets that only include verified news (BBC and NYT), our findings can inspire future studies related to the spreading of misinformation in online systems [13,49]. Our results could serve as baselines in future studies that consider the commenting dynamics of both verified and false news. Do false news trigger different patterns of impact compared to true news? Is the diffusion of false and true news governed by different fundamental mechanisms? Understanding which mechanisms play a major role in engaging users and triggering their comments might suggest intervention strategies to prevent their impact.

The collected BBC data contain several other characteristics that have not been included in the present study: comment length, comment text, as well as the number of up-votes and down-votes for each comment. Their analysis can yield further patterns in article commenting. Of particular interest is the interplay between comment sentiment and the discussion activity is of particular interest. Do positive or controversial comments help fuel the discussion? Do early emotionally loaded comments influence the long-term tone of the discussion? Which factors contribute to users approval or disapproval of a comment? Such studies can help us understand how we discuss online and how to make these discussions more construcitve.

\section{Methods}

\section{Empirical datatasets}

We regularly crawled the sport section of the BBC website (its front page and the pages dedicated to individual sports) and collected the found news articles with commenting sections. From October 1, 2018 to June 30, 2019, we collected 3,087 articles that received 852,400 comments from 67,527 readers. Each article is assigned to a sport category. The most populated categories are Football (1590 articles), Rugby Union (439 articles), Cricket (240 articles), Tennis (162 articles), Formula 1 (139 articles), Golf (123 articles) and Boxing (103 articles). Each comment is timestamped with the time resolution of $1 \mathrm{~min}$. BBC typically closes commenting on 
the second midnight after the article has been published; most of them are therefore open for $24-48 \mathrm{~h}$.

We complement the unique BBC dataset with a dataset containing articles with commenting sections from the New York Times (NYT). ${ }^{6}$ From January 1, 2017 to May 30, 2017, there are 2,801 articles that received 649,794 comments from 75,118 readers. Also here, each article is assigned to a category. Unlike for BBC, sport articles are a minority in the NYT data: The most populated categories are National (348 articles), Learning (306 articles), Magazine (262 articles), Sports (213 articles) and Foreign (204 articles). Each comment is time-stamped with the time resolution of $1 \mathrm{~min}$. While some comments arrive long after the articles are published, the median time after which the hit articles (90th percentile by the comment count) receive $99 \%$ of their comments is less than $26 \mathrm{~h}$. To study the article impact dynamics, we thus focus on the first $26 \mathrm{~h}$ of article age (the final article impact is nevertheless determined using all data). See Supplementary Information, Section S1, for detailed information about the datasets.

Both datasets can be represented as bipartite networks with user nodes and news article nodes. Each comment is then represented by a link between the user who wrote it and the news article to which the comment belongs. These networks evolve with time as the number of article nodes, user nodes, and links all gradually grow.

\section{Fitting the comment count distributions}

The maximum likelihood estimate (MLE) of the scaling parameter of the exponential distribution is known to be the sample mean, $\hat{\lambda}=\left(\sum_{i=1}^{n} c_{i}\right) / n$. As can be seen from Fig. 1, the comment count distribution follows an exponential form starting from some lower bound $\hat{c}_{\text {min }}$. The MLE estimate then changes to $\hat{\lambda}\left(\hat{c}_{\min }\right)=\left[\sum_{j}\left(c_{j}-\hat{c}_{\text {min }}\right)\right] / n\left(\hat{c}_{\text {min }}\right)$ where the summation is over $j$ for which $c_{j} \geq \hat{c}_{\text {min }}$ and $n\left(\hat{c}_{\min }\right)=\left|\left\{j: c_{j} \geq \hat{c}_{\min }\right\}\right|$ is the number of comment counts that match or exceed the lower bound. We assess the estimate uncertainty using non-parametric bootstrap — standard deviation of the MLE estimates is evaluated for 10,000 bootstrap realizations of the comment count data.

To determine $\hat{c}_{\text {min }}$, we follow the approach suggested by [11]: We choose $\hat{c}_{\text {min }}$ that minimizes the difference between the comment count distribution and the fitted exponential distribution as measured by the standard Kolmogorov-Smirnov statistic which has the form

$$
D=\max _{c \geq \hat{c}_{\min }}|S(c)-P(c)|
$$

where $S(c)$ and $P(c)$ are the cumulative distributions for the comment counts and the fitted exponential distribution, respectively. When the weighted Kolmogorov-Smirnov statistic [11] is used, which puts more weight on tails of the distributions, results do not change qualitatively. This further suggests that our fitting procedure and the conclusions drawn from the results are robust.

\footnotetext{
${ }^{6}$ Data obtained from https://www.kaggle.com/aashita/nyt-comments.
} 
The next step is to test the hypothesis that the observed comment counts indeed follow an exponential distribution. We follow again [11] where the authors suggest to use the fitted parameters to generate synthetic exponentially distributed datasets, fit each of those datasets as described above, and finally calculate the $p$ value as the fraction of synthetic datasets whose resulting $D$ exceeds that obtained for the real data.

To finally compare the statistical evidence for an exponential distribution with that for a power-law distribution, we do the same analysis for fitting a power-law distribution. Since the input data are discrete, the MLE cannot be given in a closed form [11], we numerically maximize the log-likelihood

$$
\mathcal{L}\left(\alpha, \hat{c}_{\min }\right)=-n\left(\hat{c}_{\min }\right) \zeta\left(\alpha, \hat{c}_{\min }\right)-\alpha \sum_{j: c_{j} \geq \hat{c}_{\min }} \ln c_{j} .
$$

A detailed comparison between fitting exponential and power-law distribution to the commenting data, including the log-likelihood test which directly compares the likelihood that the analyzed data has been drawn from the exponential or the power-law distribution, is presented in Sec. S2 in the SI.

Supplementary Information The online version contains supplementary material available at https://doi. org/10.1007/s42001-021-00140-w.

Acknowledgements We thank Patrick Park for stimulating discussions.

Author contributions MM conceived the study, collected and analyzed the data, and did numerical simulations. MM and MSM designed the study and wrote the manuscript. All authors have interpreted the results.

Funding Open Access funding provided by Universität Bern. This work is supported by the National Natural Science Foundation of China (Grant Nos. 11622538, 61673150, 11850410444). MSM acknowledges financial support from the URPP Social Networks at the University of Zurich, the Swiss National Science Foundation (Grant No. 200021-182659), and the UESTC professor research start-up (Grant No. ZYGX2018KYQD215). LL acknowledges the Science Strength Promotion Programme of UESTC.

Availability of data and materials Upon publication of the manuscript, the BBC commenting data and scripts to reproduce the results presented here will be made available at https://github.com/8medom/Artic le-Impact.

\section{Declarations}

Conflict of interest The authors declare that they have no competing interests.

Open Access This article is licensed under a Creative Commons Attribution 4.0 International License, which permits use, sharing, adaptation, distribution and reproduction in any medium or format, as long as you give appropriate credit to the original author(s) and the source, provide a link to the Creative Commons licence, and indicate if changes were made. The images or other third party material in this article are included in the article's Creative Commons licence, unless indicated otherwise in a credit line to the material. If material is not included in the article's Creative Commons licence and your intended use is not permitted by statutory regulation or exceeds the permitted use, you will need to obtain permission directly from the copyright holder. To view a copy of this licence, visit http://creativecommons.org/licen ses/by/4.0\%. 


\section{References}

1. Aral, S., \& Eckles, D. (2019). Protecting elections from social media manipulation. Science, 365(6456), 858-861.

2. Bandari, R., Asur, S., \& Huberman, B.A. (2012). The pulse of news in social media: Forecasting popularity. In Sixth International AAAI Conference on Weblogs and Social Media.

3. Barabási, A. L. (2018). The Formula: The Universal Laws of Success. New York Boston London: Little, Brown and Company.

4. Barnes, R. (2015). Understanding the affective investment produced through commenting on Australian alternative journalism website New Matilda. New Media \& Society, 17(5), 810-826.

5. Berger, J., \& Milkman, K. L. (2012). What makes online content viral? Journal of Marketing Research, 49(2), 192-205.

6. Broido, A. D., \& Clauset, A. (2019). Scale-free networks are rare. Nature Communications, 10(1), 1017.

7. Brossard, D. (2013). New media landscapes and the science information consumer. Proceedings of the National Academy of Sciences, 110(Supplement 3), 14096-14101.

8. Candia, C., Jara-Figueroa, C., Rodriguez-Sickert, C., Barabási, A. L., \& Hidalgo, C. A. (2019). The universal decay of collective memory and attention. Nature Human Behaviour, 3(1), 82.

9. Canter, L. (2013). The misconception of online comment threads: Content and control on local newspaper websites. Journalism Practice, 7(5), 604-619.

10. Cheng, J., Adamic, L., Dow, P.A., Kleinberg, J.M., \& Leskovec, J. (2014). Can cascades be predicted? In Proceedings of the 23rd International Conference on World Wide Web (pp. 925-936). ACM.

11. Clauset, A., Shalizi, C. R., \& Newman, M. E. (2009). Power-law distributions in empirical data. SIAM Review, 51(4), 661-703.

12. Crane, R., \& Sornette, D. (2008). Robust dynamic classes revealed by measuring the response function of a social system. Proceedings of the National Academy of Sciences, 105(41), 15649-15653.

13. Del Vicario, M., Bessi, A., Zollo, F., Petroni, F., Scala, A., Caldarelli, G., Stanley, H. E., \& Quattrociocchi, W. (2016). The spreading of misinformation online. Proceedings of the National Academy of Sciences, 113(3), 554-559.

14. Dellarocas, C., Sutanto, J., Calin, M., \& Palme, E. (2015). Attention allocation in information-rich environments: The case of news aggregators. Management Science, 62(9), 2543-2562.

15. Epstein, R., \& Robertson, R. E. (2015). The search engine manipulation effect (SEME) and its possible impact on the outcomes of elections. Proceedings of the National Academy of Sciences, 112(33), E4512-E4521.

16. Flaxman, S., Goel, S., \& Rao, J. M. (2016). Filter bubbles, echo chambers, and online news consumption. Public Opinion Quarterly, 80(S1), 298-320.

17. Fortunato, S., Bergstrom, C. T., Börner, K., Evans, J. A., Helbing, D., Milojević, S., et al. (2018). Science of science. Science, 359(6379), eaao0185.

18. Golosovsky, M. (2018). Mechanisms of complex network growth: Synthesis of the preferential attachment and fitness models. Physical Review E, 97(6), 062310.

19. Hermida, A., Fletcher, F., Korell, D., \& Logan, D. (2012). Share, like, recommend: Decoding the social media news consumer. Journalism Studies, 13(5-6), 815-824.

20. Higham, K. W., Governale, M., Jaffe, A., \& Zülicke, U. (2017). Fame and obsolescence: Disentangling growth and aging dynamics of patent citations. Physical Review E, 95(4), 042309.

21. Higham, K. W., Governale, M., Jaffe, A., \& Zülicke, U. (2019). Ex-ante measure of patent quality reveals intrinsic fitness for citation-network growth. Physical Review E, 99(6), 060301.

22. Hofman, J. M., Sharma, A., \& Watts, D. J. (2017). Prediction and explanation in social systems. Science, 355(6324), 486-488.

23. Ishii, A., Arakaki, H., Matsuda, N., Umemura, S., Urushidani, T., Yamagata, N., \& Yoshida, N. (2012). The hit phenomenon: A mathematical model of human dynamics interactions as a stochastic process. New Journal of Physics, 14(6), 063018.

24. Iyengar, S., \& Massey, D. S. (2019). Scientific communication in a post-truth society. Proceedings of the National Academy of Sciences, 116(16), 7656-7661.

25. Johnson, N. F., Velásquez, N., Restrepo, N. J., Leahy, R., Gabriel, N., El Oud, S., Zheng, M., Manrique, P., Wuchty, S., \& Lupu, Y. (2020). The online competition between pro-and anti-vaccination views. Nature, 582, 230-233.

26. Kata, A. (2012). Anti-vaccine activists, Web 2.0, and the postmodern paradigm-An overview of tactics and tropes used online by the anti-vaccination movement. Vaccine, 30(25), 3778-3789. 
27. Kong, J. S., Sarshar, N., \& Roychowdhury, V. P. (2008). Experience versus talent shapes the structure of the Web. Proceedings of the National Academy of Sciences, 105(37), 13724-13729.

28. Krapivsky, P. L., Redner, S., \& Leyvraz, F. (2000). Connectivity of growing random networks. Physical Review Letters, 85(21), 4629.

29. Ksiazek, T. B. (2018). Commenting on the news: Explaining the degree and quality of user comments on news websites. Journalism Studies, 19(5), 650-673.

30. Ksiazek, T. B., Peer, L., \& Lessard, K. (2016). User engagement with online news: Conceptualizing interactivity and exploring the relationship between online news videos and user comments. New Media \& Society, 18(3), 502-520.

31. Kwon, S., Cha, M., Jung, K., Chen, W., \& Wang, Y. (2013). Prominent features of rumor propagation in online social media. In 2013 IEEE 13th International Conference on Data Mining (pp. 1103-1108). IEEE.

32. Lazer, D. (2020). Studying human attention on the Internet. Proceedings of the National Academy of Sciences, 117(1), 21-22.

33. Lazer, D., Pentland, A., Adamic, L., Aral, S., Barabási, A. L., Brewer, D., Christakis, N., Contractor, N., Fowler, J., Gutmann, M., et al. (2009). Computational social science. Science, 323(5915), 721-723.

34. Liu, Q., Zhou, M., \& Zhao, X. (2015). Understanding News 2.0: A framework for explaining the number of comments from readers on online news. Information \& Management, 52(7), 764-776.

35. Manning, C., Raghavan, P., \& Schütze, H. (2008). Introduction to information retrieval. Cambridge University Press.

36. Medo, M., Cimini, G., \& Gualdi, S. (2011). Temporal effects in the growth of networks. Physical Review Letters, 107(23), 238701.

37. Medo, M., Zeng, A., Zhang, Y. C., \& Mariani, M. S. (2019). Optimal timescale for community detection in growing networks. New Journal of Physics, 21, 093066.

38. Parolo, P. D. B., Pan, R. K., Ghosh, R., Huberman, B. A., Kaski, K., \& Fortunato, S. (2015). Attention decay in science. Journal of Informetrics, 9(4), 734-745.

39. Radicchi, F., Fortunato, S., \& Castellano, C. (2008). Universality of citation distributions: Toward an objective measure of scientific impact. Proceedings of the National Academy of Sciences, 105(45), 17268-17272.

40. Ren, Z. M., Mariani, M. S., Zhang, Y. C., \& Medo, M. (2018). Randomizing growing networks with a time-respecting null model. Physical Review E, 97(5), 052311.

41. Salganik, M. J., Dodds, P. S., \& Watts, D. J. (2006). Experimental study of inequality and unpredictability in an artificial cultural market. Science, 311(5762), 854-856.

42. Schäfer, M.S. (2015). Digital public sphere. In G. Mazzoleni (Ed.), The International Encyclopedia of Political Communication (pp. 1-7). Wiley Online Library.

43. Shulman, B., Sharma, A., \& Cosley, D. (2016). Predictability of popularity: Gaps between prediction and understanding. In Tenth International AAAI Conference on Web and Social Media.

44. Stocking, G. (2017). Digital news fact sheet. Pew Research Center (vol. 7).

45. Szabo, G., \& Huberman, B. A. (2010). Predicting the popularity of online content. Communications of the ACM, 53(8), 80-88.

46. Thompson, D. (2017). Hit Makers. Penguin Books.

47. Tsagkias, M., Weerkamp, W., \& De Rijke, M. (2010). News comments: Exploring, modeling, and online prediction. In European Conference on Information Retrieval (pp. 191-203). Springer.

48. Valverde, S., Solé, R. V., Bedau, M. A., \& Packard, N. (2007). Topology and evolution of technology innovation networks. Physical Review E, 76(5), 056118.

49. Vosoughi, S., Roy, D., \& Aral, S. (2018). The spread of true and false news online. Science, 359(6380), 1146-1151.

50. Wang, D., Song, C., \& Barabási, A. L. (2013). Quantifying long-term scientific impact. Science, 342(6154), 127-132.

51. Wu, F., \& Huberman, B. A. (2007). Novelty and collective attention. Proceedings of the National Academy of Sciences, 104(45), 17599-17601.

52. Yang, T., Majo-Vazquez, S., Nielsen, R. K., \& González-Bailón, S. (2020). Exposure to news grows less fragmented with increase in mobile access. Available at SSRN 3564826.

53. Yucesoy, B., Wang, X., Huang, J., \& Barabási, A. L. (2018). Success in books: A big data approach to bestsellers. EPJ Data Science, 7(1), 7.

Publisher's Note Springer Nature remains neutral with regard to jurisdictional claims in published maps and institutional affiliations. 